\title{
Ethics of vaccine research
}

\author{
Christine Grady \\ Vaccination has attracted controversy at every stage of its development and use. Ethical debates should consider its \\ basic goal, which is to benefit the community at large rather than the individual.
}

$\bigvee^{a}$ ccines truly represent one of the miracles of modern science. Responsible for reducing morbidity and mortality from several formidable diseases, vaccines have made substantial contributions to global public health. Generally very safe and effective, vaccines are also an efficient and cost-effective way of preventing disease. Yet, despite their brilliant successes, vaccines have always been controversial. Concerns about the safety and untoward effects of vaccines, about disturbing the natural order, about compelling individuals to be vaccinated for the public good and the injustices of uneven access to the benefits of vaccines have been interwoven throughout the history of vaccines and remain controversial today ${ }^{1-3}$. Such controversies and the scientific complexities and successes that fuel them raise considerable ethical challenges in the development, public health use and social acceptability of vaccines. This commentary will broadly consider one aspect of these ethical challenges in the clinical testing of vaccines.

Vaccine development is a lengthy, expensive and multifaceted process of basic and clinical research, production, licensing and marketing. Clinical testing, usually with large numbers of healthy subjects, is an integral and essential part of the process. Principles, codes and norms of ethics guiding the ethical conduct of all clinical research apply to vaccine research, yet most such guidance concentrates on therapeutic clinical trials of interventions for individuals seeking treatment. Little specific attention has been given to how the ethics of vaccine trials differ.

Applying a framework for ethical research One framework for ethical clinical research synthesizes and simplifies guidance found in existing codes and regulations and describes seven principles universally applicable to ethical clinical research ${ }^{4}$. The seven principles

Christine Grady is in the Department of Clinical Bioethics, Warren Grant Magnuson Clinical Center, National Institutes of Health, Building 10/1C118, Bethesda, Maryland 20892-1156, USA.

e-mail: cgrady@nih.gov include value, validity, fair subject selection, favorable risk/benefit ratio, independent review, informed consent and respect for enrolled participants. Applying these principles to vaccine research allows consideration of some of the particular challenges inherent in testing vaccines (Box 1).

Ethically salient features of clinical vaccine research include the fact that it involves healthy subjects, often (or ultimately) children and usually (at least when testing efficacy) in very large numbers. Furthermore, in vaccine research, individuals are asked to accept risk for the public good and the prospect of 'provisional' benefit. Finding safe and effective vaccines benefits the public health through reducing the burden of disease among individual members of the public and indirectly protecting unvaccinated members of the public through 'herd immunity'. Individual benefit is 'provisional', however, because individuals benefit directly from investigational vaccines only if they are sufficiently exposed to the infectious agent at some future time, had received the active vaccine and had been sufficiently protected.

\section{Value}

According to the framework, the first ethical requirement is that the research question have potential social or scientific value to justify exposing individuals to research risks and inconvenience. Research that can improve health or increase useful knowledge has value. Determining the value of a vaccine trial calls for an understanding that "...the major purpose is to determine whether a vaccine is of use as a public health tool. This is in contrast to the many large scale therapeutic trials where the objective is to determine what is best for individuals. Yet, our ethical system is built around individuality..."5.

Although vaccines have considerable public health value, it does not necessarily follow that every vaccine research proposal has social or scientific value. A specific vaccine study or program's value depends on its contribution to the goal of finding a safe, effective and available vaccine useful within the context in which it will be used and acceptable to those who will use it. This assessment considers details about the public health need (such as the prevalence, burden and natural history of the disease, as well as existing strategies to prevent or control it), the scientific data and possibilities (preclinical and clinical data, expected mechanism of action and immune correlates) and the likely use of the vaccine (who will use and benefit from it, safety, cost, distribution, political will, acceptability and so on). An important consideration is the value of the research for those participating in the vaccine testing and how it will be maximized through dissemination of knowledge gained, product development, long-term research collaboration and/or health system improvements. No judgment of value is straightforward or immune from criticism, however, as illustrated by the following example in which the value of vaccine research was in dispute. An effective rotavirus vaccine was pulled from the US market because of a high incidence of intussusception in vaccinated children. Consequently, debates ensued about proceeding with large trials planned in developing countries where disease burden and vaccine efficacy might differ. Debates highlighted differing risk-benefit calculations in developing countries in which childhood deaths from rotavirus were high, as well as how acceptance of vaccine might be compromised by the US decision ${ }^{6,7}$. Assessing the value of this research was clearly, and appropriately, context dependent.

\section{Validity}

A valuable research question ethically requires a valid research design and implementation. Carefully chosen and rigorous study design, methodology and implementation strategies appropriate to the research question, and likely to provide interpretable generalizable data, also are balanced by considerations of fairness and minimizing risk. In ensuring that the scientific design of a vaccine trial realizes social value for the primary beneficiaries, several controversial design and methodological issues can arise. 


\section{BOX 1 FRAMEWORK APPLIED TO VACCINE RESEARCH}

\author{
Elements of ethical research \\ Value
}

Validity

Fair subject selection
Independent review

Informed consent
Respect for enrolled participants

\section{Specific considerations}

- Public health need

- Scientific possibility

- Social acceptability

- Political will

- Endpoints and measurement of efficacy

- Choice of control (such as placebo)

- Randomization

- Feasibility of recruitment and follow-up

Favorable risk/benefit ratio

- Large numbers of healthy subjects, often including children

- Participants in developing countries

- Risks to individuals: physical, social, confidentiality, future trials

- Risk of no protection

- Provisional benefit to individual

- Benefits to community and public good

- Fair benefit evaluations

- Familiarity with vaccine research

- International settings

- Misconceptions about vaccines

- Cultural and social differences

- Community permission

- Monitoring welfare
- Right to withdraw

- Treatment and/or compensation for vaccine-induced injury

Carefully defined endpoints, for example, are essential for high-quality science as well as ethical science. Although some vaccines prevent infection, many alter the course of infection and have their protective effect on clinical disease. In vaccine trials, surrogate endpoints can sometimes be accurately used to measure efficacy, but more often clinical endpoints are necessary. A vaccine trial evaluating clinical outcomes requires considerable time, resources and careful planning for statistical power and long-term follow-up. Furthermore, apparent conflicts can arise between the interventional care of those who become infected and the measurement of critical clinical endpoints. For a placebocontrolled trial of an experimental vaccine against tuberculosis in bacillus CalmetteGuerin-naive people, for example, a decision about purified protein derivative testing and prophylactic treatment of those found positive would have to be balanced against the need to determine the efficacy of the vaccine against clinical disease ${ }^{8}$. Shorter trials using surrogate endpoints to measure outcomes are attractive, but can in some cases, provide misleading results.

Challenge studies in which volunteers are deliberately infected with a microbe can serve many important scientific and public health purposes, addressing questions about etiology, pathogenicity, pathogenesis, immune response and protection ${ }^{9}$. Although challenge studies can be scientifically valuable and efficient, there is something disquieting about deliberately infecting someone, potentially causing considerable discomfort in the process. Thus, microbes amenable to challenge studies are carefully selected when the rationale is strong, the risk is low and symptoms or effects of the challenge are selflimiting or reversible or can be treated easily. The voluntary informed consent of the volunteer is also essential.

The choice of an appropriate control in any randomized clinical trial, including vaccine trials, can be contentious. Tension can exist between the need to ensure that the design realizes the scientific objectives while still guaranteeing research participants the health care interventions to which they are entitled. A placebo control is acceptable when no effective vaccine is available, although it is ethically important to integrate other known preventive strategies, such as health education, into both arms of a vaccine trial. When a partially effective vaccine is available or a known effective vaccine is used in some places but not others, the justification for and choice of a control can be more difficult. The use of placebo controls in one arm of National Institutes of Health-sponsored acellular pertussis vaccine trials in Sweden and Italy, for example, was criticized despite a previous decision by the Swedish and Italian governments not to routinely use the available whole killed pertussis vaccine ${ }^{10}$. These trials could not have been done in the US, where pertussis vaccine is required for children to go to school.

Randomization, a powerful research tool used to balance comparison groups, is a common feature of vaccine trials. Randomization raises ethical questions about participant autonomy, especially as empirical data show that randomization is poorly understood by research subjects. Although most phase 3 vaccine efficacy trials randomize individuals to demonstrate the direct protective effects of vaccine, some studies use cluster randomization or randomization by community or group. Although often justified by the need to evaluate herd immunity and indirect as well as direct protection from a vaccine, community randomization could be seen as jeopardizing the autonomy rights of individuals within randomized communities.

Ethically, it is necessary to ensure that a research study is feasible given the social, political and cultural environment. In this regard, a feasible strategy for recruiting a large sample and following them over time is essential.

\section{Fair subject selection}

Fairness in subject selection is realized when subjects are chosen mainly because of their scientific appropriateness for a study, balanced by considerations of risk, benefit and vulnerability. Fairness in the processes and outcomes of subject selection prevent exploitation of vulnerable individuals and populations. Scientifically, those most appropriate for vaccine efficacy studies are populations with a sufficient and predictable incidence of the disease in question to be able to show the effect of the vaccine. The sample size needed to demonstrate vaccine efficacy is usually large and is calculated in part on expected incidence, taking into account previous and evolving incidence of infection, demographics of the target population and characteristics of those who are likely to volunteer. Historically, vaccines were often tested in 'vulnerable' and captive populations, such as prisoners and the institutionalized mentally impaired. At present, inclusion of these vulnerable groups is restricted by specific and protective regulations. 
Considerations of who might benefit from the vaccine are central to subject selection. The World Medical Association Declaration of Helsinki states that "Medical research is only justified if there is a reasonable likelihood that the populations in which the research is carried out stand to benefit from the results of the research" (Ethical principles for medical research involving human subjects; http://www.wma.net/e/policy/b3.htm). This has important implications when developing country populations are involved in vaccine research.

Some have argued that phase 1 vaccine studies for diseases or conditions prevalent in the developing world, such as malaria or HIV infection, should be undertaken first in the sponsor's country, to minimize the possibility of exploiting vulnerable populations in resource-poor countries ${ }^{11}$. Thinking is shifting, however, because studies should be responsive to the health needs of populations and there are good reasons to bring vaccine trials, even at early stages, to populations that will benefit most. On the other hand, communities may be vulnerable to exploitation if fair benefits from the research are not negotiated ${ }^{12}$.

Vaccine trials eventually enroll large numbers of healthy children or infants because many vaccines are ultimately used in children. The amount of research risk to which children can be exposed without corresponding benefit is limited by regulation in USsponsored research. Because children cannot protect their own interests through informed consent, parents and guardians are asked for permission to enroll their children in vaccine research. Investigators and institutional review boards (IRBs) should ensure that parents and guardians are well informed and make decisions compatible with the interests of the child. In hepatitis A vaccine trials in northern Thailand, parents gave permission for their children to have both hepatitis A and hepatitis $B$ vaccine as part of the trial in a cross-over design ${ }^{13}$.

\section{Risks and benefits}

A favorable risk/benefit ratio exists when risks are justified by benefits and research is designed so that risks are minimized and benefits to subjects and society are maximized. In vaccine research, most risk accrues to individual participants and benefits accrue mainly to the community in finding a safe and protective vaccine. Individuals may receive provisional or future benefit from vaccine trials, but most benefit only indirectly. A utilitarian calculation justifies both the testing and use of vaccines, accepting risk of harm to a few for the benefit of the many. For example, 40,000 children were vaccinated in the hepatitis A trial mentioned above; efficacy was determined based on 38 cases of hepatitis A in the control group and 2 in the vaccine group. Perhaps a clearer utilitarian justification exists for research with the transmission-blocking vaccine for malaria. Individuals given only the transmission-blocking vaccine would not themselves be protected from malaria, but would interrupt the transmission cycle, protecting others in close contact with them ${ }^{14}$. Infectious challenge studies are another example of individuals accepting some risk for benefit to society and no expected benefit to themselves.

An ethical requirement of all clinical research is to minimize risk and maximize benefit. In HIV vaccine trials, for example, careful counseling and education about risk reduction strategies are an essential part of minimizing risk, as participants may incorrectly assume they are protected by an experimental vaccine and may actually increase risky behavior that exposes them to HIV.

\section{Independent review}

IRBs or research ethics committees with varied expertise and no personal or business interests in the research evaluate said research for adherence to established ethical guidelines. However, there are certain issues that may make review of vaccine studies more difficult. Some IRBs may simply lack expertise related to the science of vaccines and the structure of vaccine studies such as challenge studies or 50,000person efficacy field trials. Similarly, because many vaccine trials involve populations in developing countries, IRBs need information and sensitivity to both the context in which a study will be done and current guidance and controversies in the ethics of international research.

\section{Informed consent}

Once a proposal is deemed valuable, valid and acceptable with respect to risks, benefits and subject selection, individuals are recruited and asked for informed consent. Although widely valued, informed consent is imperfectly realized in clinical research. Because massive public education campaigns promote the use of vaccines for public health, individuals may not appreciate how experimental vaccines differ in the context of research. Two other important aspects of vaccine trials may be difficult for individuals to understand: randomization and determination of vaccine efficacy. Studies of informed consent that have measured participant understanding in clinical trials have consistently demonstrated poor comprehension of randomization and placebo design ${ }^{15}$. Although little is known about the degree to which participants understand that some of them or their children will (must) get the disease or infection to prove efficacy, this knowledge is likely to be low.

Most agree that individual informed consent is necessary for vaccine trial participation, even when community permission is indicated ${ }^{16}$. Some studies use a 'staged consent' that may include seeking permission from community leaders, providing information to community members through group meetings or public media, providing more detailed information to interested individuals, allowing time for deliberation or consultation with family or health care providers and ultimately obtaining voluntary authorization from the person to be vaccinated (or their parent or guardian). Information should be disclosed in culturally and linguistically appropriate ways, and creative strategies for educating participants about vaccine trials are often warranted, especially in populations unfamiliar with research or with high rates of illiteracy. Another debated issue is the extent to which participants in vaccine trials should be compensated for their participation. Because attitudes about compensation vary considerably, it is important to involve the local community in establishing recruitment procedures and compensation schemes. When an entire community is randomized to receive an investigational vaccine, strategies for upholding the right of individuals to refuse participation or to withdraw from the research while 'saving face' should be sought.

\section{Respect for enrolled participants}

Research participants deserve continued respect, through continuous monitoring of their welfare, maintaining confidentiality of private information, allowing withdrawal without penalty and assuring access to successful interventions or new information generated by the study. Large vaccine efficacy trials often include a cross-over design or other mechanism for ensuring that the control group receives vaccine if it is found to be protective. Less settled are questions regarding the circumstances, if any, in which study participants can ethically remain untreated after they become infected. Although this was heavily debated in a series of global meetings sponsored by UNAIDS, no consensus was reached for HIV vaccine trials by the time the UNAIDS guidelines were published. Although most agree there is need to ensure 
that vaccine trial participants who become infected with HIV will receive treatment, the challenge is determining how this should happen ${ }^{17}$. Providing treatment and compensation for vaccine-related injury is consistent with respect for the vaccinee. In the US, the National Vaccine Injury Compensation Program provides compensation for certain injuries deemed related to licensed childhood vaccines, but no such mechanism exists for injuries sustained in clinical trials of vaccines.

\section{Conclusion}

A framework for ethical research is helpful in identifying ethical challenges common to all research as well as those unique to vaccine trials. Careful attention to ethical issues is essential to the successful development of vaccines as invaluable public health interventions.

\section{ACKNOWLEDGMENTS}

The views expressed here are the author's and do not necessarily reflect those of the Clinical Center, the National Institutes of Health, the Public

Health Service or the Department of Health and Human Services.

1. Spier, R.E. Vaccine 16, 1788-1794 (1998),

2. Macklin, R. \& Greenwood, B. in The Vaccine Book (eds. Bloom, B. \& Lambert, P.) 119-127 (Elsevier Science, San Diego, 2003).

3. Ulmer, J.B. \& Liu, M. Nat. Rev. Immunol. 2, 291-294 (2002).

4. Emanuel, E., Wendler, D. \& Grady, C. J. Am. Med. Assoc. 283, 2701-2711 (2000).
5. Hall, A. Int. J. Tuberc. Lung Dis. 3, 745 (1999).

6. Weijer, C. Br. Med. J. 321, 525-526 (2000)

7. Melton, L. Lancet 356, 318 (2000).

8. Snider, D.E. Clin. Inf. Dis. 30, S271-S275 (2000)

9. Levine, M.M. Dev. Biol. Stand. 95, 169-174 (1998).

10. Edwards, K. \& Decker, M. N. Eng. J. Med. 334, 391-392 (1996).

11. Macklin, R. \& Greenwood, B. in The Vaccine Book (eds. Bloom, B. \& Lambert, P.) 123 (Elsevier Science, San Diego, 2003).

12. Participants in the 2001 Conference on Ethical Aspects of Research in Developing Countries. Science 298, 2133-2134 (2002).

13. Innis, B. et al. J. Am. Med. Assoc. 271, 1363-1364 (1994).

14. Hoffman, S. \& Richie, T. in The Vaccine Book (eds. Bloom, B. \& Lambert, P.) 291-306 (Elsevier Science, San Diego, 2003).

15. Leach, A. et al. Soc. Sci. Med. 48, 139-149 (1999).

16. UNAIDS. Ethical considerations in HIV preventive vaccines (UNAIDS, Geneva, 2000).

17. Berkley, S. Lancet 362, 992 (2003). 